\title{
La opinión del profesorado de Educación Secundaria sobre el papel del patrimonio en la enseñanza formal de las ciencias sociales: un estudio de caso $^{1}$
}

\section{The opinion of secondary school teachers on the role of heritage in the formal education of Social Sciences: a case study}

\author{
Sebastián MOLINA PUCHE y Rafael Emilio MUÑOZ CUTILLAS \\ Universidad de Murcia
}

Recibido: Febrero 2015

Evaluado: Abril 2015

Aceptado: Abril 2015

\section{Resumen}

Este artículo investiga el uso que los profesores de ciencias sociales dan a los diferentes elementos que constituyen el patrimonio cultural para enseñar historia. Para ello, partimos de un estudio de caso realizado sobre el Departamento de Geografía e Historia del IES Azorín, radicado en Yecla (Murcia). La finalidad de este estudio es determinar las metodologías, perspectivas y valoraciones que realiza el profesorado de estos elementos patrimoniales, así como su introducción en el proceso de enseñanza y aprendizaje de las ciencias sociales. Esta investigación está organizada a partir de una serie de entrevistas semiestructuradas donde nos centramos en reconocer las estrategias, apreciaciones y propuestas de mejora planteadas por los profesores del centro.

Palabras clave: educación media superior, patrimonio, metodología de aula, didáctica del patrimonio, didáctica de la historia.

\begin{abstract}
The paper analyzes how social science educators make use of cultural heritage resources in teaching history. For a case study, we have selected the Department of Geography and History of the Azorín Secondary Education Institute, located in Yecla (Murcia, Spain). The purpose of this study is to determine the methodologies, perspectives and evaluations of cultural heritage elements, carried out by teachers, as well as their insertion in the teaching and learning process of the social sciences. This research is organized from a series of semi-structured interviews, and we focus on recognizing the strategies, insights and suggestions for improvement raised by the teachers at the secondary education institute.
\end{abstract}

\footnotetext{
${ }^{1}$ Este trabajo es resultado del proyecto de investigación "La formación en identidad regional en ciencias sociales a partir del patrimonio inmaterial. Aplicaciones didácticas para Educación Secundaria Obligatoria" (referencia 18951/JLI/13), financiado por Fundación Séneca, Agencia Regional de Ciencia y Tecnología de la CARM.
} 
Keywords: secondary education, heritage, classroom methodology, didactics of heritage, didactics of history.

Dentro de la construcción del carácter comprometido, socioeducativo y crítico del ejercicio del educador, la confluencia del binomio patrimonio-educación formal, con sus impedimentos y sus beneficios, es uno de los aspectos más valorados en el panorama educativo español, pero de los menos analizados a partir de experiencias "reales". Pese a la enorme consideración que se suele otorgar al patrimonio como recurso y objeto educativo (y especialmente en el ámbito de las ciencias sociales), son muy pocos los estudios -merece la pena destacar el de Estepa, Ávila y Ferreras, 2013que se han centrado en intentar descubrir qué lugar ocupa lo patrimonial en las aulas, y sobre todo, hasta qué punto es un referente para el profesorado. A fin de intentar dar respuesta a esta problemática, hemos iniciado un proyecto de investigación, financiado por la Fundación Séneca de la Comunidad Autónoma de la Región de Murcia (proyecto 18951/JLI/13), cuya finalidad es conocer hasta qué punto y de qué manera se utiliza el patrimonio cultural en la enseñanza de la materia de Ciencias Sociales de Educación Secundaria, qué función tiene o puede tener en la configuración de identidades colectivas del alumnado, y qué opinión tiene el profesorado de esta materia sobre su utilidad como recurso didáctico en la dinámica habitual del aula de Geografía e Historia. Es en el marco de esta investigación en la que se encuentra el trabajo que aquí presentamos: la primera tarea a realizar en el citado proyecto fue la realización de un trabajo de investigación preliminar a partir de un estudio de caso (los componentes del departamento de ciencias sociales de un instituto de Educación Secundaria situado en Yecla, una localidad del norte de la provincia), cuyo análisis ha servido para la realización de un cuestionario al profesorado de la región que se va a convertir en una de las principales herramientas del citado proyecto de investigación. Pese a tratarse de una investigación inicial y de un ejemplo concreto, los resultados obtenidos en las entrevistas semiestructuradas realizadas han sido lo suficientemente reveladores como para poder ser considerados merecedores de ser dados a conocer: es más que probable que los datos obtenidos con la encuesta no hagan más que ratificar las conclusiones ofrecidas en este estudio preliminar, de ahí su interés.

\section{Marco teórico}

Como muy bien señala González Monfort (2006), la investigación en Didáctica de las Ciencias Sociales (en adelante, DCS) pretende comprender la realidad educativa de estas materias, saber qué ocurre y por qué ocurre cuando se enseñan y se aprenden contenidos geográficos, históricos y sociales con el objetivo de transformar el ejercicio docente hacia prácticas educativas innovadoras y eficientes. Este proceso de investigación de los procesos didácticos ha permitido definir las finalidades de la enseñanza de las ciencias sociales (en adelante, CCSS) en tres objetivos imprescindibles: comprender a los seres humanos de sociedades próximas y lejanas en el tiempo y/o el espacio, formar ciudadanos y lograr el desarrollo personal del alumnado. Con estas grandes finalidades, está fuera de toda duda el enorme valor que 
tiene el patrimonio como herramienta educativa capaz de cumplir estos preceptos básicos. De hecho, García, Jiménez y Moreno (2003), Calaf (2010) y Fontal (2011), sitúan el uso del patrimonio como epicentro para la adquisición de conceptos propios de esta disciplina como evolución y memoria histórica, las nociones de cambio y permanencia o la asunción de la interrelación de factores multicausales que explican transformaciones sociales.

Este potencial educativo del patrimonio, que parte de las propuestas de Pluckrose (1993) de valorar el patrimonio como un articulador del conocimiento histórico-social, ha supuesto una reinterpretación de la idea del patrimonio (Ortuño, Gómez y Molina, 2011): traspasar la concepción tradicional del patrimonio como algo estático y cuyo único valor es descriptivo, hacia una valoración dinámica, abierta e integral, que muestre su naturaleza como construcción social históricamente mutable, una representación simbólica de identidades sociales, políticas y religiosas.

En suma, la inserción de la Didáctica del Patrimonio debería incluirse en el proceso educativo, no como un fin en sí mismo (Cuenca, 2002; Calaf, 2009) sino como un pretexto, no sólo para la difusión de las CCSS, sino como una herramienta de comunicación para descubrir "quién soy y quiénes son los otros" (González Monfort, 2011, 62), potenciándose de esta manera el papel de la educación patrimonial en la construcción de identidades sociales (Yanes, 2013).

Por lo tanto, de la misma manera, el carácter binómico del conjunto patrimonio educación, proporciona al centro educativo la capacidad de ser un mediador entre patrimonio y la sociedad, siendo valorado como una de las instituciones más importantes para ello, dada su estructura y los medios de que dispone, así como los fuertes procesos de socialización que convergen en su interior (González y Pagés, 2005, 64).

Sin embargo, frente a estas propuestas de uso, tradicionalmente las relaciones entre patrimonio y enseñanza han sido dispares y poco claras (Hernández Cardona, 2003, 445). En efecto, en el campo de la educación formal se ha reaccionado de forma tardía, no se ha aprovechado su potencialidad, y aún hoy la relación con el patrimonio es una importante carencia, algo puramente testimonial, que se queda en un conjunto de buenas intenciones escasamente cumplidas (Fernández Salinas, 2005; Rico y Ávila, 2003). En contraposición, han tomado ventaja los ámbitos de la educación no formal relacionados con elementos patrimoniales (turismo cultural, proyectos interpretativos, programas museológicos, etc.) que contemplan diferentes finalidades, metodologías y objetivos (Estepa, Ávila y Ruiz Fernández, 2007).

Este traspaso de responsabilidades desde la educación formal a la informal (e incluso la no formal) se observa perfectamente en el curriculum educativo español y en los libros de texto. En el caso del currículo, aunque debido a la delimitación del espacio no podemos realizar un estudio exhaustivo de la inclusión del patrimonio en el mismo, este tema ha sido tratado por autores como Fernández Casildo (2008), y especialmente Cuenca (2003) y Fontal (2011). González Monfort (2011) habla de una presencia del patrimonio en el curriculum "escasa, puntual y poco coherente" (p. 68) Para Cuenca (2002), la finalidad crítica y comprometida se ve devaluada, 
convirtiéndose en "un mero objeto artístico" (p. 261). Para Rico y Ávila (2003), su inclusión no es más que una simple declaración de intenciones, una oportunidad desaprovechada. En conclusión, una perspectiva bastante nefasta para la posible inclusión en el aula pues, si no se restituye la importancia del patrimonio en la propia ley educativa, la utilización por parte del profesorado descansa únicamente de su propia voluntad y deseo.

En lo referente a la presencia de lo patrimonial en los libros de texto de CCSS, estos materiales, que siguen monopolizando el tratamiento de los contenidos en el aula (Cuenca, Estepa y Martín, 2011, 54), han recibido una especial atención por parte del grupo EDIPATRI de la Universidad de Huelva. Así, Estepa, Ferreras, López Cruz y Morón (2011) hacen un sistemático y preciso análisis de los manuales de diferentes editoriales mediante un riguroso sistema de parámetros, llegando a conclusiones interesantes: el patrimonio se utiliza como un mero ejemplo histórico-artístico, con una escasa visión interdisciplinar, siendo superados sus valores sociales, ciudadanos y críticos por una esencia basada en la imagen y en la anécdota reducida muchas veces a simples imágenes de apoyo, perdiendo su potencialidad crítica y reflexiva.

A ello se puede sumar lo que se ha dado en llamar la "epistemología práctica del docente" (Domínguez, 2003, 232), una suerte de malas experiencias y pocas ilusiones que hacen desfallecer las ganas de incluir metodologías activas en el aulas, manteniendo la tradicional articulación de la clase sobre la palabra del profesor, el soporte papel y el alumno pasivo. Esta enseñanza tradicional, con escasa valoración de los contenidos de tipo procedimental o actitudinal, basada en una comprensión de la historia cronológica, memorística y conceptual (Monteagudo y Villa, 2011) supone un grave obstáculo para la inclusión de lo patrimonial en las aulas (Cuenca, 2002; Domínguez, 2003; Ávila y Mattozzi, 2009). La mayoría de obstáculos radican en las carencias a la hora de comprender el concepto de patrimonio, contemplándose una visión muy fragmentaria y académica, lo que induce a que prevalezca una perspectiva monumentalista del patrimonio basada en un enfoque descriptivo-memorístico pasivo. Por otro lado, nos encontramos un cierto inmovilismo, una falta palpable de propiciar la participación del alumnado que -si ya de por sí es renuente- dicha estructuración bastante cerrada y dirigida, no está abierta a su intervención y debate. Unas dificultades similares son las que encontramos cuando analizamos las nociones y actitudes del profesorado en formación: Cuenca y Domínguez (2002) y Cuenca et al. (2011) realizaron diversas investigaciones al respecto, demostrando que, en la formación inicial del profesorado, no se aborda el patrimonio como un referente clave para la DCS. La perspectiva de los alumnos se centra en una visión en base a su antigüedad o su grandiosidad, es decir, su componente histórico-artístico, siendo muy poco valorado su carácter simbólico-identitario.

Todo ello ha llevado a que, en términos generales, la educación informal, e incluso la no formal, haya "tomado ventaja", en lo que al aprovechamiento del patrimonio se refiere. Y es la explicación de que las preguntas que formulan Cuenca et al. (2011) sean totalmente pertinentes:

si el patrimonio es tan motivador, ¿por qué suele ser tan aburrido para el alumnado? Si el patrimonio tiene tal potencial educativo, ¿por qué se trabaja 
poco en las aulas? Si es tan atractivo, ¿por qué se mantiene tan poco contacto directo? (p. 55).

Para intentar dar respuesta a estas preguntas hemos iniciado el anteriormente citado proyecto de investigación del cual, como hemos indicado anteriormente, presentamos en estas páginas las primeras etapas de la investigación y, con ellas, las primeras conclusiones parciales.

\section{Marco empírico}

Como hemos señalado anteriormente, esta investigación inicial se centra en el análisis del uso del patrimonio y la historia local que realizan los profesores que conforman el Departamento de CCSS del IES José Martínez Ruiz "Azorín” de Yecla (Murcia), con el fin de, a través del análisis de este caso concreto, intentar dilucidar las debilidades, fortalezas y oportunidades derivadas de su aplicación en el proceso de enseñanza-aprendizaje. Evidentemente, debido a las propias características de la muestra utilizada en este estudio, el objetivo de este trabajo no es más que realizar un primer acercamiento, siquiera superficial, a esta problemática, cuya principal pretensión no es otra que servir de inicio a una investigación más amplia. No obstante, es cierto que al hacer uso de una metodología de investigación cualitativa basada en el estudio de caso, los datos y conclusiones obtenidas aportan cierta significatividad perse.

\section{Objetivos}

Los objetivos generales del proyecto en el que se inserta esta investigación inicial (y que, por lo tanto, comparte) son los siguientes:

1. Conocer la percepción de los profesores de la materia de Ciencias Sociales, Geografía e Historia de ESO sobre el valor del patrimonio local para la enseñanza de la Historia.

1.1. Identificar el uso didáctico del patrimonio local que realizan en los profesores de CCSS en sus clases.

2. Valorar el potencial del patrimonio local como "contenedor" de la historia.

2.1. Reconocer propuestas de uso del patrimonio local en el aula de Historia

El primer objetivo general está dirigido a reconocer la perspectiva del profesorado respecto al uso del patrimonio local como estrategia de acercamiento entre el alumno y la materia. Por ello, a través del objetivo específico, analizaremos el uso didáctico que hacen de este recurso en sus clases y las implicaciones metodológicas que les aporta. Tras éste, el segundo objetivo se centra en la valoración de este material patrimonial como herramienta en la transmisión de conocimientos en las clases de CCSS. Ello 
conlleva un objetivo específico dirigido a la concreción de metodologías, estrategias y herramientas para su uso en el aula.

\section{Metodología}

Los propósitos señalados anteriormente podrían alcanzarse, en términos de investigación, a través de distintos diseños metodológicos. Mientras que algunas estrategias buscan un análisis predictivo y de control, evitan valorar los significados sino que buscan identificar rutinas e irregularidades. Otras metodologías gestionan el estudio de los fenómenos y procesos que caracterizan la vida en el aula, buscando dotar de sentido aspectos estudiados en la interacción de los alumnos y la institución. Sin embargo, dado el carácter inicial de esta investigación, hemos optado por un estudio descriptivo con metodología cualitativa a partir del análisis de los datos obtenidos en entrevistas semiestructuradas cuyas preguntas han sido validadas por jueces-experto. Nos hemos decidido por la metodología cualitativa dado que precisábamos un acceso más profundo a conocimientos, experiencias y prácticas del desarrollo de los procesos educativos.

\section{Muestra}

La concreción de la muestra para esta primera investigación fue seleccionada mediante un muestreo deliberado al pretender llevar a cabo un estudio de caso. Así, nos decidimos por valorar la perspectiva de trabajo y metodología de uso de los profesores de un departamento de CCSS ya que, siguiendo el marco teórico, pretendemos contestar a una evidencia teórica mediante el ejercicio del método científico (Cardona, 2007; Albert, 2007).

La muestra se corresponde con los profesores miembros del departamento de CCSS del IES “Azorín” de Yecla (Murcia). La elección del centro y, sobre todo, de la localidad, fue totalmente intencionada: con algo más de treinta mil habitantes, Yecla ${ }^{2}$ es una población "media" dentro del conjunto de la Comunidad Autónoma que, para los objetivos de este estudio, presenta unas características muy interesantes, pues en el término municipal y el caso urbano podemos encontrar multitud de elementos patrimoniales tanto de carácter natural como cultural $^{3}$, además de contar con un interesante y recientemente reformado museo municipal ${ }^{4}$. En cuanto al instituto, se encuentra situado en el centro histórico de la ciudad, siendo el primer instituto que se fundó en la ciudad (actualmente hay tres en la población), originado a partir del anterior Instituto Nacional de Bachillerato que surgió de la laicización del antiguo Colegio de Escolapios. Este instituto imparte Educación Secundaria y también

\footnotetext{
${ }^{2}$ Con 34.601 habitantes (Censo de 2013), Yecla es el noveno municipio por población.

${ }^{3}$ A destacar, por ejemplo, las pinturas rupestres del monte Arabí, pertenecientes al Arte Levantino (8000 A.C.); el yacimiento romano de Los Torrejones (s. I-VI); el yacimiento hispanomusulmán de Yakka (s. XI-XIII); la Iglesia de la Asunción (s. XV); la plaza mayor (s. XV-XVII); casas solariegas de los siglos XVI a XVIII; la basílica de la Purísima (s. XVIII-XIX), edificios modernistas, etc.

A este respecto, ver http://www.yecla.es/CIUDAD/Turismo/VisitasdeInteresporYecla.aspx

${ }^{4}$ El museo arqueológico Cayetano de Mergelina. Ver http://museoarqueologicodeyecla.org/
} 
Bachilleratos, siendo el único que desarrolla el bachillerato de arte en la comarca. Esta situación geográfica del centro trae consigo una amplia diversidad de alumnos con condiciones socioeconómicas diferentes.

Dicho departamento, que constituye la muestra analizada, está compuesto por ocho docentes, licenciados en Geografía e Historia, siete hombres y una mujer (aunque consideramos que la variable género no influye en la materia de estudio), con una media de edad en torno a los 45 años. A continuación, aportamos una tabla (Tabla 1) elaborada a partir de una ficha de identificación recogida en el cuestionario, donde se engloban los principales datos útiles de esta muestra:

\begin{tabular}{lcccccc}
\hline & Sexo/edad & $\begin{array}{c}\text { Años en } \\
\text { activo }\end{array}$ & $\begin{array}{c}\text { Años en } \\
\text { el IES } \\
\text { Azorín }\end{array}$ & $\begin{array}{c}\text { Años docente } \\
\text { en Secundaria }\end{array}$ & $\begin{array}{c}\text { iParticipa en } \\
\text { proyectos de } \\
\text { innovación? }\end{array}$ & $\begin{array}{c}\text { Nivel } \\
\text { académico }\end{array}$ \\
\hline Sujeto 1 & $\mathrm{H} / 34$ & 6 & 2 & 6 & $\mathrm{Si}$ & Licenciado \\
\hline Sujeto 2 & $\mathrm{H} / 48$ & 24 & 12 & 22 & $\mathrm{Si}$ & Licenciado \\
\hline Sujeto 3 & $\mathrm{M} / 36$ & 12 & 10 & 12 & $\mathrm{No}$ & Licenciado \\
\hline Sujeto 4 & $\mathrm{H} / 36$ & 9 & 6 & 9 & $\mathrm{Si}$ & Licenciado \\
\hline Sujeto 5 & $\mathrm{H} / 58$ & 35 & 34 & 24 & $\mathrm{Si}$ & Licenciado \\
\hline Sujeto 6 & $\mathrm{H} / 58$ & 36 & 17 & 17 & $\mathrm{Si}$ & Licenciado \\
\hline Sujeto 7 & $\mathrm{H} / 51$ & 8 & 8 & 8 & $\mathrm{No}$ & Licenciado \\
\hline Sujeto 8 & $\mathrm{H} / 37$ & 8 & 4 & 9 & $\mathrm{Si}$ & Licenciado \\
\hline & Tabla 1. Datos de identificación de los sujetos entrevistados &
\end{tabular}

En términos generales, consideramos que la muestra es lo suficientemente diversa para otorgar a los datos que ofrecen cierta representatividad: profesores con una amplia experiencia docente junto a otros que no aún no llevan diez años en la profesión; docentes que han participado activamente en proyectos de innovación educativa (lo que puede denotar interés por mejorar su práctica educativa) frente a otros que no lo han hecho; y profesores que siempre han impartido clase en Educación Secundaria junto a otros que proceden de centros de Formación Profesional.

\section{Instrumentos}

Para la recogida de la información decidimos apoyarnos en la realización de entrevistas semiestructuradas, ya que por su planteamiento más abierto (un guión de preguntas flexibles) dan pié a ampliar los registros de técnicas, opiniones, consejos y experiencias de los entrevistados. Para el diseño de las preguntas, centramos la orientación de la entrevista hacia la comprensión de tres bloques de conocimiento relacionados con los objetivos propuestos en la investigación y que permitían estructurar su posterior comprensión mediante el sistema de categorías (Tabla 2). Así, contamos con un primer bloque basado en el análisis de la valoración del patrimonio local para la práctica docente. Un segundo bloque sobre el uso didáctico que se realiza 
del patrimonio dentro del aula. Y un tercer bloque sobre propuestas para aumentar el uso del patrimonio en clase.

\begin{tabular}{ll}
\hline Bloque & Preguntas \\
\hline I. Valoración del & 1. ¿Consideras importante el uso del patrimonio en las clases de CCSS? ¿Por qué? \\
\cline { 2 - 3 } & $\begin{array}{l}\text { 5. ¿Tiene cabida la identidad local en tus clases? ¿Crees que esta actitud se desarrolla } \\
\text { en las clases de Secundaria? ¿Tus alumnos te preguntan por aspectos del patrimonio } \\
\text { local? }\end{array}$ \\
\hline II. Uso didáctico & $\begin{array}{l}\text { 2. Hemos detectado en la bibliografía que los elementos patrimoniales son utilizados } \\
\text { de tres modos, como contenidos conceptuales (Prehistoria); contenidos actitudinales }\end{array}$ \\
& $\begin{array}{l}\text { (identidad - mediación cultural); y en menor medida como contenidos } \\
\text { procedimentales (observación, análisis, comparación) ¿es alguno de estos tu caso? }\end{array}$ \\
\cline { 2 - 3 } & $\begin{array}{l}\text { 3. Es perceptible que existe un aislamiento de la historia local en las materias de } \\
\text { CCSS. Para ti, ¿funciona de forma integrada o es un bloque al margen del desarrollo? }\end{array}$ \\
& $\begin{array}{l}\text { Es decir, ¿el patrimonio local y por tanto la historia local es un recurso didáctico o un } \\
\text { elemento anecdótico? }\end{array}$ \\
\cline { 2 - 3 } & $\begin{array}{l}\text { 4. ¿Por qué crees que el patrimonio se trabaja fundamentalmente a través de salidas e } \\
\text { itinerarios didácticos, por tanto, de forma excepcional; y no de forma continuada en la } \\
\text { programación de aula habitual? ¿Has visitado con tus alumnos espacios patrimoniales } \\
\text { locales? (Museo Arqueológico / yacimientos locales / espacios relacionados con la } \\
\text { historia local) Sí/no ¿Por qué?). }\end{array}$ \\
\hline $\begin{array}{l}\text { 6. ¿Cómo podríamos presentar los elementos patrimoniales para que no sólo sea un } \\
\text { recurso didáctico para trabajar otros contenidos, sino también un objeto de estudio por } \\
\text { sí mismo? }\end{array}$ \\
$\begin{array}{l}\text { 7. ¿Qué herramientas precisarías para poder realizar una mejor utilización del } \\
\text { patrimonio local en tus aulas? }\end{array}$ \\
\hline III. Propuestas
\end{tabular}

Tabla 2. Preguntas realizadas a los entrevistados

\section{Resultado y discusión}

Tras la transcripción de la grabación de las entrevistas y el análisis de contenido para poder identificar categorías o referentes en base a los cuales realizar el estudio, obtuvimos 3 categorías, 8 subcategorías y 23 sub-subcategorías que resumimos en la Tabla 3 .

\begin{tabular}{|c|c|c|c|c|}
\hline \multirow{6}{*}{ 氙 } & \multirow{6}{*}{ 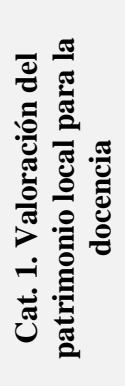 } & \multirow{6}{*}{$\begin{array}{l}\text { 1.1 Importancia } \\
\text { en el proceso de } \\
\text { enseñanza- } \\
\text { aprendizaje }\end{array}$} & \multirow{2}{*}{$\begin{array}{l}\text { 1.1.1 Alta } \\
{[\mathrm{s} 2-\mathrm{s} 6]}\end{array}$} & $\begin{array}{l}\text { 1.1.1.1 Estrategia de acercamiento a la materia } \\
\text { [s2-s6] }\end{array}$ \\
\hline & & & & 1.1.1.2 Favorece el trabajo en el aula [s6] \\
\hline & & & \multirow{2}{*}{ 1.1.2 Media [s1-s3-s5] } & $\begin{array}{l}\text { 1.1.2.1 Relacionar la materia con el entorno del } \\
\text { alumno [s1-s3] }\end{array}$ \\
\hline & & & & $\begin{array}{l}\text { 1.1.2.2 Materialización de conceptos abstractos } \\
\text { [s3-s5] }\end{array}$ \\
\hline & & & \multirow{2}{*}{$\begin{array}{l}\text { 1.1.3 Relativa [s4-s7s- } \\
\text { 8] }\end{array}$} & $\begin{array}{l}\text { 1.1.3.1 Complejidad en relacionar conceptos } \\
\text { generales y elementos concretos [s4] }\end{array}$ \\
\hline & & & & 1.1.3.2 Demostrar una utilidad clara y accesible \\
\hline
\end{tabular}




\begin{tabular}{|c|c|c|c|c|}
\hline & & & & para el alumno [s4-s7] \\
\hline & & & & 1.1.3.3 Ausencia en el libro de texto [s7s8] \\
\hline & & 1.2.1 Habi & al: prevalece & u carácter histórico-artístico [s2-s6] \\
\hline & 1.2 Uso durante & 1.2.2 Anec & tico: materi & complementario [s4-s7-s5] \\
\hline & el ejercicio & $1.2 .3 \mathrm{No} \mathrm{e}$ & a en la & 1.2.3.1 Por falta de tiempo [s1-s8] \\
\hline & & $\begin{array}{l}\text { programac } \\
{[\mathrm{s} 1-\mathrm{s} 3-\mathrm{s} 8]}\end{array}$ & n de aula & $\begin{array}{l}\text { 1.2.3.2 Carencia de estrategias de integración } \\
\text { [s1-s3] }\end{array}$ \\
\hline & & 1.3.1 Com & contenido & $\begin{array}{l}\text { 1.3.1.1 Utilizado por su valor ejemplificador } \\
\text { [s2-s6] }\end{array}$ \\
\hline & & $\begin{array}{l}\text { conceptua } \\
\text { s4-s5-s6-s }\end{array}$ & 8] & $\begin{array}{l}\text { 1.3.1.2 Menos utilizado por sus características } \\
\text { específicas [s8] }\end{array}$ \\
\hline & & 1.3.2 Com & contenido & $\begin{array}{l}\text { 1.3.2.1 Utilizado como estrategia de aplicación } \\
\text { de conocimientos generales [s1-s4-s6] }\end{array}$ \\
\hline & & $\begin{array}{l}\text { procedime } \\
\text { s4-s6-s8] }\end{array}$ & al [s1-s2- & $\begin{array}{l}\text { 1.3.2.1 Menos utilizado por las experiencias } \\
\text { insatisfactorias a la hora de aplicar } \\
\text { metodologías investigativas [s5] }\end{array}$ \\
\hline & $\begin{array}{l}\text { 1.J Metoaologia } \\
\text { de uso }\end{array}$ & 1.3.3 Com & contenido & $\begin{array}{l}\text { 1.3.3.1 Utilizado por su valor emocional para } \\
\text { motivar su preservación y conservación [s6-s8] }\end{array}$ \\
\hline & & actitudinal & 1-s2-s6-s8] & $\begin{array}{l}\text { 1.3.3.2 Menos utilizado por su interés } \\
\text { extracurricular [s2] }\end{array}$ \\
\hline & & 1.3.4 En sa & & $\begin{array}{l}\text { 1.3.4.1 Destacan sus beneficios: permite una } \\
\text { didáctica diferente al trascender la normalidad } \\
\text { del aula [s1-s2-s7] }\end{array}$ \\
\hline & & extraescol & [s1-s2-s7] & $\begin{array}{l}\text { 1.3.4.2 Destacan sus inconvenientes: la } \\
\text { planificación al principio del curso, a falta de } \\
\text { conocer al grupo [s5-s6-s8] }\end{array}$ \\
\hline & & 2.1.1 Alta & loración par & el proceso educativo [s2-s6] \\
\hline & & 2.1.2 Med & por la falta & tiempo para su desarrollo [s5] \\
\hline$\frac{\pi}{\bar{E}}$ & $\begin{array}{l}2.1 \text { Importancia } \\
\text { para el }\end{array}$ & & & $\begin{array}{l}\text { 2.1.3.1 El profesor no es de origen local [s1-s3- } \\
\text { s8] }\end{array}$ \\
\hline$\frac{\pi}{\pi}$ & & $\begin{array}{l}\text { 2.1.3 Ваја } \\
\text { s3-s4-s7-s }\end{array}$ & nula [S1- & $\begin{array}{l}\text { 2.1.3.2 El temario a desarrollar es demasiado } \\
\text { denso [s1-s3s-s4-s8] }\end{array}$ \\
\hline$\stackrel{\mathscr{J}}{\varrho}$ & & & & 2.1.3.3 Falta de interés en su uso [s8] \\
\hline$\stackrel{\varrho}{ٍ}$ & & 2.2.1 Inter & potenciado & través del profesor [s2-s6] \\
\hline$\stackrel{\ominus}{E}$ & $\begin{array}{l}\text { para el } \\
\text { alumnado }\end{array}$ & $\begin{array}{l}2.2 .2 \text { Inter } \\
\text { s7] }\end{array}$ & escaso, redu & ido a los principales ámbitos patrimoniales [s4- \\
\hline$\underline{\tilde{z}}$ & & 2.2.3 Sin $\mathrm{i}$ & erés [s1-s3-s & $-88]$ \\
\hline تृ & & & & 2.3.1 Como recurso didáctico [s2-s4-s6] \\
\hline 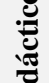 & & 2.3.1 En e & & $\begin{array}{l}\text { 2.3.2 Como elemento anecdótico [s1-s3-s5-s7- } \\
\text { s8] }\end{array}$ \\
\hline 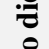 & 23 Mothá & & & 2.3.2.1.1 Museo Arqueológico local [s2-s5] \\
\hline$\underline{n}$ & de uso & $\begin{array}{l}2.3 .2 \\
\text { Visitas a }\end{array}$ & $\begin{array}{l}2.3 .2 .1 \\
\text { Fsnacio }\end{array}$ & 2.3.2.1.2 Conjunto Monumental [s2-s5-s7-s8] \\
\hline$\stackrel{\sim}{ \pm}$ & & espacios & $\begin{array}{l}\text { Espacio } \\
\text { visitado }\end{array}$ & 2.3.2.1.3 Monte Arabí (Arte Rupestre) [s5] \\
\hline 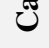 & & patrimoni & & 2.3.2.1.4 Yacimientos arqueológicos [Ninguno] \\
\hline & & & $\begin{array}{l}2.3 .2 .2 \\
\text { Trabas }\end{array}$ & $\begin{array}{l}\text { 2.3.2.2.1 Falta de facilidades de las entidades } \\
\text { gestoras [s4-s6-s7] }\end{array}$ \\
\hline
\end{tabular}




\begin{tabular}{|c|c|c|c|}
\hline & & & $\begin{array}{l}\text { 2.3.2.2.2 Temprana resolución de estos } \\
\text { contenidos. [s5-s8] }\end{array}$ \\
\hline \multirow{12}{*}{ 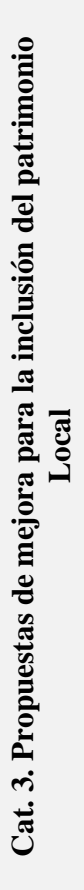 } & \multirow{8}{*}{$\begin{array}{l}3.1 \text { A nivel } \\
\text { interno del IES }\end{array}$} & \multirow{3}{*}{$\begin{array}{l}\text { 3.1.1 Mejora de las } \\
\text { estrategias burocráticas }\end{array}$} & $\begin{array}{l}\text { 3.1.1.1 Compromiso en la PGA con la } \\
\text { valoración de esta materia [s3] }\end{array}$ \\
\hline & & & $\begin{array}{l}\text { 3.1.1.2 Organización de materias específicas } \\
\text { (optativas, desdobles, seminarios) [s4-s6-s7] }\end{array}$ \\
\hline & & & $\begin{array}{l}\text { 3.1.1.3 Organización de salidas con carácter } \\
\text { transversal [s3-s5-s7] }\end{array}$ \\
\hline & & \multirow{4}{*}{$\begin{array}{l}\text { 3.1.2 Nuevos } \\
\text { materiales didácticos: } \\
\text { trabajar desde los } \\
\text { centros de interés del } \\
\text { alumno }\end{array}$} & 3.1.2.1 De carácter divulgativo [s1-s2-s3-s8] \\
\hline & & & 3.1.2.2 De carácter pedagógico [s4] \\
\hline & & & $\begin{array}{l}\text { 3.1.2.3 Adaptados al nivel cognitivo del } \\
\text { alumnado al que se dirige [s5-s6] }\end{array}$ \\
\hline & & & $\begin{array}{l}\text { 3.1.2.4 Compendios científicos sobre } \mathrm{H}^{\mathrm{a}} \text { Local } \\
\text { [s8] }\end{array}$ \\
\hline & & \multicolumn{2}{|c|}{$\begin{array}{l}\text { 3.1.3 Generar una base de datos digitalizada con materiales patrimoniales } \\
\text { [s1-s2-s3-s4-s6-s7] }\end{array}$} \\
\hline & \multirow{4}{*}{$\begin{array}{l}3.2 \text { A nivel } \\
\text { externo al IES }\end{array}$} & \multicolumn{2}{|c|}{ 3.2.1 Formación del profesorado en Historia y Patrimonio Local [s4-s8] } \\
\hline & & \multirow{3}{*}{$\begin{array}{l}\text { 3.2.2 Mayor } \\
\text { implicación de las } \\
\text { instancias municipales } \\
\text { [s3-s5-s6-s7] }\end{array}$} & 3.2.2.1 Flexibilidad de horarios [s4] \\
\hline & & & 3.2.2.2 Asistencia al aula [s5-s6] \\
\hline & & & $\begin{array}{l}\text { 3.2.2.3 Elaboración de visitas guiadas basadas } \\
\text { en el currículum académico [s } 3 \text {-s5-s6-s7] }\end{array}$ \\
\hline
\end{tabular}

Tabla 3. Categorías halladas en las entrevistas

A continuación, procederemos a hacer un somero análisis de cada subcategoría con el fin de profundizar en los aspectos recogidos producto de las entrevistas.

\section{Categoría 1. Valoración del patrimonio local para la práctica docente}

\section{Importancia del proceso de enseñanza-aprendizaje}

La importancia otorgada al patrimonio es de un nivel medio, centrando su uso en su carácter material: la plasmación de los conceptos abstractos desarrollados en la materia. Sin embargo, el $25 \%$ de los sujetos reiteran su valor como estrategia de acercamiento al alumno mientras que el $37,5 \%$ presentan reticencias a la hora de utilizarlos, centradas en su escasa presencia en los libros de texto, su utilidad para el ejercicio docente o destacando las singularidades de unos elementos concretos frente a la seriaciones de características. El sujeto 4 nos ofrece un interesante ejemplo de respuesta:

Supone la realidad material de los conceptos abstractos explicados, un refuerzo de los conceptos generales trabajados. [...] Debe ser accesible, visualmente claro y debe tener una utilidad clara para poder incluirlo en mis clases; que lo singular, lo local, no confunda la explicación general. [S4] 


\section{Uso durante el ejercicio docente}

El uso del patrimonio local es muy reducido, tan sólo el 25\% de la muestra lo utilizan habitualmente, aunque realizan una gran defensa del mismo, siguiendo la tónica de la importancia que, al menos desde los años ochenta, se le ha dado a la localidad tanto en los currículos escolares como a los libros de texto de ciencias sociales (González García, 2011). Por contra, un 37'5\% lo consideran un material complementario, mientras que el otro $37^{\prime} 5 \%$ reconoce no utilizarlo, justificando esta ausencia en la amplitud de la programación junto a la ausencia de técnicas de uso. A este respecto el Sujeto 3 declara:

Es complicado. Al final, de manera realista, lo utilizas de manera anecdótica. Pero no es que nosotros no queramos, es porque no tenemos tiempo para dedicarle y colocarlo en el temario con un valor equiparable al resto de temas. De hecho, si tú miras las programaciones, las referencias a historia local o tal son anecdóticas o directamente ni aparecen. Por eso, se convierten en "zancadillas", en problemillas a la hora de dirigir la ejecución del temario... por eso al final se quedan en anécdotas, en recursos didácticos para explicar otros hechos y conceptos. [S3]

\section{Metodología de uso}

Dentro de las técnicas de uso, respecto a las clasificaciones propuestas en el marco teórico observamos cómo la totalidad de la muestra destaca el uso conceptual del patrimonio local destacando su valor ejemplificador pero cuestionando su especificidad. Con respecto a su uso como contenido procedimental, el 62 ' $5 \%$ lo destaca como una forma de aplicar los conocimientos generales al entorno cercano. Sin embargo, nos encontramos como el $12{ }^{\prime} 5 \%$ destacan haber aplicado metodologías investigativas con el alumnado sin lograr los frutos deseados. En su valor como contenido actitudinal, el $37^{\prime} 5 \%$ considera que lo utiliza en este sentido, buscando un interés conservacionista principalmente. Además, en relación con el patrimonio, el $37^{\prime} 5 \%$ destaca la salida extraescolar como una metodología de uso diferente a la del aula, aunque deben estar planificadas antes del inicio del curso, con la impersonalidad que ello provoca. Como ejemplos de respuesta: "[...] conceptual, suelo utilizarlo como ejemplo de lo que explico"(s3); “[...] Trabajas actitudes y procedimientos principalmente en las salidas, en clase, utilizo el patrimonio de modo conceptual"(s2); o "[...] Mi interés procedimental se basa en que se fijen en edificios y sepan resaltar las características generales explicadas"”(s4).

\section{Categoría 2. Uso didáctico del patrimonio local en el aula}

\section{Importancia para el profesorado}

A este respecto, frente al $25 \%$ de la muestra que lo valora muy positivamente, el 62 ' $5 \%$ le otorga un valor bajo o nulo, centrando sus justificaciones en la densidad del 
temario $(50 \%)$, el hecho de no ser oriundo o no vivir en Yecla $\left(37^{\prime} 5 \%\right)$ o un directo desinterés en su uso para el ejercicio didáctico (12’5\%). El sujeto 8 declara:

[...] Tiene cabida, como ya te digo, pero muy poca, sobre todo por la densidad del temario. [...] También te digo que hay parte de desconocimiento por mi lado. Yo no soy de Yecla y, aunque me he informado de muchas cosas sobre la ciudad, tampoco creo que tenga un conocimiento lo suficientemente científico... pero ya te digo que es más porque no tengo tiempo. Por ello, muchas veces he pensado en prepararme algo, pero es que sé que al final no lo voy a poder utilizar. [S8]

\section{Importancia para el alumnado}

Esta categoría se basa en las interpretaciones que obtienen los profesores en el contacto directo con el alumnado. Así, aunque no es una evaluación directa de la opinión de los alumnos, nos parece interesante para inferir la comprensión del profesorado de elementos del proceso educativo. En este caso, el $50 \%$ considera que el alumnado no tiene ningún tipo de interés al respecto del patrimonio local, mientras que un $25 \%$ destaca que al tratar ciertos temas como prehistoria o etapa musulmana sí que preguntan sobre la influencia local. Sin embargo, otro $25 \%$ piensa que si se potencia por parte del profesor, los alumnos buscan mantener la línea de conocimientos al respecto de la materia local. El sujeto 3 declara:

No, nunca, no se me ocurre que me hayan preguntado al nada al respecto... A menos que se lo hayas mostrado tú, lo que les gusta, y te prestan más atención, pero de ellos no nace la curiosidad de preguntarte [...] Y, como decía antes, si no se les ha atraído o interesado por el tema, ¿cómo van a preguntar al respecto? [S3]

\section{Metodología de uso}

Por un lado, dentro de la metodología de uso del patrimonio local en el aula, pretendíamos establecer una perspectiva muy clara: delimitar si estaba integrado o era un mero añadido. A este respecto, el $62{ }^{\prime} 5 \%$ lo definían como un elemento anecdótico, utilizado para rebrotar el interés o la atención hacia lo explicado. Sin embargo, el $37{ }^{\prime} 5 \%$ lo destaca como un recurso didáctico, incluso utilizado como protagonista en sus clases. Respecto a las salidas, nos encontramos con que ningún entrevistado había visitado con sus alumnos los yacimientos arqueológicos locales, aumentando este porcentaje en lo referido al Monte Arabí (12’5\%), el museo arqueológico local (25\%) y la visita al conjunto monumental (50\%). Al respecto del bajo número de estas visitas, los entrevistados destacaron que los problemas residían en la configuración de los temas a tratar en estos espacios respecto al currículum (25\%) así como ciertas dificultades a la hora de concertar y desarrollar las visitas (37'5\%). Como ejemplo de respuesta: 
Tratar el temario es un trabajo complejo, cuesta mucho que los alumnos se queden con las ideas generales. En teoría, lo ideal sería ver la Historia con todos los niveles en paralelo: el nivel general, el local... en la práctica, prefiero insistir en las ideas generales y cuando la historia local puede ayudar a ilustrar la introduzco. No es lo ideal, pero entre que los alumnos no tienen mucha base de ninguno de los dos niveles y que hay que dar muchas cosas... [S5]

\section{Categoría 3. Propuestas de mejora para la inclusión del patrimonio local}

\section{A nivel interno del IES}

Frente a la evaluación y análisis del uso del patrimonio local, propusimos la configuración de unas propuestas de mejora con la intención de proponer medidas capaces de paliar las deficiencias observadas. En este caso, estas mejoras se buscarían a nivel interno al centro. De ello, el $75 \%$ de los entrevistados valorarían muy positivamente disponer de una base de datos digitalizada con información al respecto de materiales patrimoniales (fotografías, gráficos, definiciones, etc.). Además proponen la generación de nuevos materiales didácticos pero, aunque no es tan uniforme el carácter de estos materiales, todos están de acuerdo en que deben ser recursos del profesor, no para entregar directamente al alumno. Sin embargo, el aspecto que más destaca es el requerimiento de mayor profundidad de la historia local dentro de la Programación General Anual (PGA). Así, un 12'5\% solicita su tratamiento comprometido dentro de la PGA, mientras que un 37'5\% reclaman la organización de materias específicas al respecto y otro $37^{\prime} 5 \%$ pide la configuración de salidas extraescolares de carácter transversal para propiciar un tratamiento integral del espacio visitado:

No es tanto cuestión de tener necesidad de recursos materiales; materiales hay muchos a fin de cuentas, lo que pasa es que sobre patrimonio local los materiales de los que disponemos son exageradamente específicos: monográficos, publicaciones concretísimas, artículos muy científicos... Demasiado especializado, con un sentido más científico que divulgativo. [S2]

\section{A nivel externo del IES}

Dentro de estas propuestas de mejora, también incluíamos un apartado sobre cómo propiciar la visita a los espacios patrimoniales locales. A este respecto, el $25 \%$ cree indispensable la generación de cursos sobre historia de Yecla para profesores, como herramienta fundamental para iniciar el interés por esta materia. Por otro lado, al ser cuestionados sobre este tema, el 50\% destacó la necesidad de una mayor implicación de las instancias municipales, solicitando una flexibilización de los horarios (12'5\%), una mayor asistencia al aula (25\%) y sobre todo (50\%) la elaboración de un programa de visitas guiadas basadas en el currículum académico. Como señala el sujeto 3:

[Para utilizar el patrimonio local] lo primero, conocer bien qué se ofrece en la ciudad, a nivel histórico, artístico y natural. Lo segundo, tener una oferta que lo 
haga interesante. Lo tercero, más facilidades para sacarlos de aquí, que organizar una salida es muy difícil. [...] si te soy sincera, creo que es importante que los trabajadores del museo o directamente de la concejalía, no sé, quien controle el tema patrimonial en la ciudad; vengan a los institutos a "ofertar" sus materiales. Puede sonar frívolo, pero deberían planteárselo desde un punto de vista más comercial: tú tienes un producto y tienes que venderlo a quién pueda estar interesado en comprártelo. [S3]

\section{Conclusión}

Si el patrimonio tiene tal potencial educativo... ¿Por qué se trabaja tan poco en las aulas? A partir de esta cuestión planteada por Cuenca et al. (2011, 55), que recogíamos en el marco teórico, hemos desarrollado un proyecto de investigación que arranca con un estudio de caso que nos ha permitido obtener datos realmente interesantes. Hemos procedido a determinar cómo una institución educativa pública, regida por unas normas y un programa educativo general se hace cargo de una de las competencias que debe cumplir como espacio formador: construir ciudadanos con las habilidades democráticas suficientes como para valorar su entorno de forma crítica y contextualizada y más responsable con su legado cultural y con su historia (Yanes, 2013). En este caso, cómo se gestiona la historia y el patrimonio local en las clases de CCSS desde la perspectiva técnica del profesorado de estas materias.

Sin embargo, nuestra valoración, desde una perspectiva transversal de la investigación, no es del todo positiva. Frente al potencial educativo del patrimonio reconocido en la bibliografía, creemos que todavía queda mucho trabajo para integrar estos contenidos dentro de los procesos didácticos. Como hemos visto, frente a esa concepción dinámica, abierta e integral de la que parten los autores del corpus teórico, los profesores entrevistados todavía participan de una visión fragmentaria y académica del patrimonio, otorgándole una valoración más estática y monumentalista, con una introducción dentro del proceso educativo en un carácter descriptivo y, por tanto, pasivo (algo que ya observó Domínguez, 2003). Pero ante todo, no queremos culpar a los profesores de esta práctica, creemos que su labor es verdaderamente loable dentro de la vorágine que representa el trabajo diario dentro del aula, cada día con más alumnos y menos tiempo para dedicarlo a mejorar la aplicación de conocimientos a partir de procesos innovadores. Además, hemos podido observar que existe inquietud por darle uso al entorno próximo al centro, acusando una falta de medios y de unos materiales didácticamente consecuentes. Desde nuestro punto de vista, creemos que la clara actitud devaluadora presente en los libros de texto respecto al patrimonio visto de forma general junto a la falta de implicación de los legisladores nacionales y autonómicos trae consigo -a través de un curriculum que abandona estos elementos que dan lugar a la historia- una depreciación de estos contenidos dentro de la programación general del centro y del propio Departamento. Además, la falta de implicación activa de las administraciones públicas gestoras de estos espacios patrimoniales con la formación del alumnado, con su falta de presencia en el centro y en la proposición de proyectos interdidácticos con el profesorado, es un acicate más para el abandono de estos contenidos. Esta cadena de devaluaciones creemos que genera un efecto negativo 
en la docencia: los profesores ya deben hacer frente a demasiadas dificultades a diario, convirtiéndose en un escollo para la generación de una verdadera cultura escolar implicada con su entorno.

Y es que, desde nuestro punto de vista, esta ausencia del patrimonio y la historia local está provocando que la enseñanza de las CCSS -y en especial de la historia-, se quede a medio camino; es decir, que los objetivos más a largo plazo que conlleva la asimilación de estos contenidos no se están llevando a cabo satisfactoriamente. Así, la ausencia de la historia y el patrimonio local destruye esa idea de que la educación es una práctica social, situada y contextualizada, perdiéndose esa concepción de que el entorno es un producto histórico. La ausencia del espacio cercano dentro del ejercicio educativo no permite al alumno reconocer que lo que aprende tiene que ver con sus vivencias y sus experiencias, con la historia vital de su comunidad; sino que sólo es un contenido teórico más. Además, este carácter teórico es el mismo que se observa en el aula, ya que el trabajo del historiador no se refrenda dentro del proceso educativo, tan sólo una simple transmisión de conocimientos, un ejercicio docente demasiado tradicional para la juventud del siglo XXI.

Esta nueva generación que hoy se educa en aulas como las del IES Azorín compondrá la sociedad civil que en un futuro deberá determinar, en su fuero interior, en una concepción personal y subjetiva determinada por su condición cultural, económica y por sus experiencias vitales; la que decidirá reconocer o no estos bienes culturales y apoyar proyectos de conservación de los mismos. Y creemos firmemente que eso no va a ser posible si en la etapa vital que determina su madurez -la adolescencia- y gestiona el desarrollo de los procesos cognitivos a través de la educación recibida, no se lleva a cabo un proceso de asimilación de las raíces históricas de su comunidad y su entorno a través de sus restos culturales.

Nuestra propuesta para resolver esta deficiencia parte de la resituación del proceso comunicativo: pensar la educación como una práctica social situada y contextualizada, devolviéndonos así al pensamiento original de que la relación que un individuo mantiene con su cultura dependerá fundamentalmente de las condiciones en que las ha adquirido. Esta visión refuerza la inclusión del patrimonio cultural en las materias relacionadas con las áreas sociales de la educación, mediante la revalorización del entorno como estrategia para adquirir una mirada integral y crítica al territorio como producto histórico, propiciando así el protagonismo del patrimonio local como recurso de las estrategias educativas innovadoras.

Desde nuestra perspectiva, la revalorización holística de los entornos próximos al centro educativo permite una reorganización del proceso de enseñanza-aprendizaje a partir de la reflexión, la observación y la experiencia propia, aspectos que demuestran la significatividad del uso de estos materiales patrimoniales locales. Una escuela relacionada con su entorno suma dos principios a su ejercicio formativo: por un lado, acomete un proceso de renaturalización de los procesos, una reminiscencia a los llamados "métodos naturales" que ya expusieran Freinet o Dewey (Calaf, 2009) como estrategia para asentar los conocimientos y no convertirlos en meros conceptos alejados, poco prácticos y de adquisición efímera. Por otro lado, permite una recontextualización de los contenidos, propiciando el uso de metodologías activas: el 
uso del patrimonio local a través de una metodología investigativa-participativa permitiría desarrollar actividades interdisciplinares, experienciales, donde el aprendizaje se transformara en duradero y funcional mediante la apertura al medio cercano. Un ejercicio puramente constructivista de creación de conocimiento.

\section{Referencias bibliográficas}

ALBERT, M. J. (2007). La investigación educativa: claves teóricas. Madrid: Mc GrawHill.

ÁVILA, R. M. (2005). Reflexiones sobre la enseñanza y el aprendizaje del patrimonio integrado. Una experiencia en la formación de maestros. Revista Investigación en la Escuela, 5, 43-53.

ÁVILA, R. M. Y MATTOZZI, I. (2009). La Didáctica del Patrimonio y la educación para la ciudadanía. En Ávila, R. M., Borghi, B. y Matozzi, I. (coord.). La Educación de la Ciudadanía y la Formación del Profesorado (pp. 327-362). Bologna: Pátron.

CALAF, R. (2009). Didáctica del Patrimonio. Gijón: Editorial TREA.

CALAF, R. (2010). Un modelo de investigación en Didáctica del Patrimonio que recupera la práctica profesional en didáctica de las ciencias sociales. Revista Enseñanza de las Ciencias Sociales, 9, 17-28.

CARDONA, M. C. (2002). Introducción a los métodos de investigación en Educación. Madrid: Editorial EOS.

CUENCA, J. M. (2002). El patrimonio en la Didáctica de las Ciencias Sociales. Análisis de concepciones, dificultades y obstáculos para su integración en la enseñanza obligatoria. Tesis Doctoral. Universidad de Huelva.

CUENCA, J. M. (2003). Análisis de concepciones sobre la enseñanza del patrimonio en la educación obligatoria. Revista Enseñanza de las Ciencias Sociales, 2, 37-45.

CUENCA, J. M. Y DOMÍNGUEZ, C. (2002). Análisis de concepciones sobre la enseñanza del patrimonio en la formación inicial del profesorado de Educación Secundaria. En Estepa, J., de la Calle, M. y Sánchez, M. (edit.). Nuevos horizontes en la formación del profesorado en las Ciencias Sociales (pp. 79-96). Palencia: AUPDCS.

CUENCA, J. M. Y ESTEPA, J. (2003). El patrimonio en las ciencias sociales. Concepciones transmitidas por los libros de texto de ESO. En Ballesteros, E. et al. (coord.). El patrimonio y la didáctica de las ciencias sociales (pp. 91-102). Cuenca: AUPDCS.

CUENCA, J. M., ESTEPA, J., Y MARTÍN, M. (2011). El patrimonio cultural en la educación reglada. Patrimonio Cultural de España. Patrimonio y Educación, 5, $45-58$ 
DOMÍNGUEZ, C. (2003). El patrimonio. A la búsqueda de un lugar en el currículo. En Ballesteros, E. et al. (coord.). El patrimonio y la didáctica de las ciencias sociales (pp. 225-234). Cuenca: AUPDCS.

ESTEPA, J., ÁVILA, R. M. Y RUIZ, R. (2007). Concepciones sobre la enseñanza y difusión del patrimonio en las instituciones educativas y los centros de interpretación. Estudio descriptivo. Revista Enseñanza de las Ciencias Sociales, 6, 75-94.

ESTEPA, J., ÁVILA, R. M. Y FERRERAS, M. (2013). Concepciones del profesorado de Primaria y Secundaria acerca del patrimonio y su enseñanza y aprendizaje. En Estepa, J. (coord.). La educación patrimonial en la escuela y el museo: investigación y experiencias (pp. 41-59). Huelva: Universidad de Huelva.

ESTEPA, J., FERRERAS, M., LÓPEZ CRUZ, I., Y MORÓN, H. (2011). Análisis del patrimonio presente en los libros de texto. Obstáculos, dificultades y propuestas. Revista de Educación, 355, 573-589.

FERNÁNDEZ CASILDO, M. (2008). La utilización del patrimonio cultural como recurso didáctico en la enseñanza secundaria, al tiempo que como vía de conocimiento y valoración de uno mismo. En Pereiro, X., Prado, S. y Takenaka, H. (edit.). Educación e interpretación. Cruzando límites y produciendo alternativas (pp. 109-124). San Sebastián: Ankulegi.

FERNÁNDEZ SALINAS, V. (2005). Finalidades del patrimonio en la educación. Revista Investigación en la escuela, 5, 7-18.

FERRERAS, M. Y ESTEPA, J. (2011). Análisis y evaluación de libros de texto de conocimiento del medio desde la Didáctica del Patrimonio. En Miralles, P., Molina Puche, S. y Santisteban, A. (edit.). La evaluación en el proceso de enseñanza y aprendizaje de las ciencias sociales (pp. 87-98). Murcia: AUPDCS.

FONTAL, O. (2007). El patrimonio cultural del entorno próximo. Un diseño de sensibilización para secundaria. Revista Enseñanza de las Ciencias Sociales, 6, 3147.

FONTAL, O. (2011). El patrimonio en el marco curricular español. Patrimonio Cultural de España. Patrimonio y Educación, 5, 21-44.

GARCÍA, C., JIMÉNEZ, M. D. Y MORENO, C. (2003). El patrimonio arqueológico y su tratamiento en la enseñanza. En Ballesteros, E. et al. (coord.). El patrimonio y la didáctica de las ciencias sociales (pp. 443-454). Cuenca: AUPDCS.

GONZÁLEZ GARCÍA, E. (2011). La localidad y los imaginarios sociales en el Segundo Ciclo de Educación primaria en los años 80: una muestra representativa. Cabás, 6.

GONZÁLEZ MONFORT, N. (2006). L'ús didàctic i el valor educatiu del patrimoni cultural. (Tesis Doctoral). Universidad Autònoma de Barcelona. 
GONZÁLEZ MONFORT, N. (2011). La presencia del patrimonio cultural en los currículos de educación infantil, primaria y secundaria obligatoria de España. Patrimonio Cultural de España. Patrimonio y Educación, 5, 59-74.

GONZÁLEZ MONFORT, N. y PAGÉS, J. (2005). La presencia del patrimonio cultural en los libros de texto de la ESO en Cataluña. Revista Investigación en la Escuela, 5, 55-66.

HERNÁNDEZ CARDONA, F. X. (2003). El patrimonio como recurso de la enseñanza de las ciencias sociales. En Ballesteros, E. et al. (coord.). El patrimonio y la didáctica de las ciencias sociales (pp. 455-466). Cuenca: AUPDCS.

MONTEAGUDO, J. Y VILLA, J. (2011). La evaluación de las competencias básicas en la materia de Historia en $4^{\circ}$ de ESO en la Región de Murcia. En Miralles, P. et al. (edit.). La evaluación en el proceso de enseñanza y aprendizaje de las ciencias sociales (pp. 317-326). Murcia: AUPDCS.

PLUCKROSE, H. (1993). Enseñanza y aprendizaje de la Historia. Madrid: Morata.

RICO, L. Y ÁVILA, R. M. (2003). Difusión del Patrimonio y Educación. El papel de los materiales curriculares. Un análisis crítico. En Ballesteros, E. et al. (coord.). El patrimonio y la didáctica de las ciencias sociales (pp. 31-40). Cuenca: AUPDCS.

YANES CABRERA, C. (2013). Nuevos contextos de educación y representación de la cultura popular y de las minorías étnicas: los museos y su función educadora. Cabás, 10, 29-42.

\section{Correspondencia con los autores}

Sebastián MOLINA PUCHE

Dpto. de Didáctica de las Ciencias Matemáticas y Sociales

Facultad de Educación. Universidad de Murcia

Campus de Espinardo, CP 30100. Murcia (España)

e-mail: smolina@um.es

Rafael Emilio MUÑOZ CUTILLAS

Dpto. de Didáctica de las Ciencias Matemáticas y Sociales

Facultad de Educación. Universidad de Murcia

Campus de Espinardo, CP 30100. Murcia (España)

e-mail: rafaelemilio.munoz@um.es 